\title{
THE DYNAMICS OF A DIFFUSING GAS*
}

BY HENRI PUTMAN (Universite Laval, Quebec)

By use of a hydrodynamical approach, Stefan ${ }^{1}$ derived the following equation for the diffusion of two gases:

$$
\rho_{1} \xi_{1}=-\frac{\partial p_{1}}{\partial x}-A_{12 \rho_{1} \rho_{2}\left(u_{1}-u_{2}\right)} .
$$

Here $p_{1}$ is the partial pressure of the first gas, $p_{1}$ is its density, $u_{1}, \xi_{1}$ are respectively the $x$-components of the velocity and acceleration of one of its particles, $u_{2}, \rho_{2}$ refer to the second gas, and $A_{12}$ is a constant. There are two other equations similar to (1) corresponding to the $y$ and $z$-components, and a further set of three equations for the second gas.

Equation (1) is a simplified form of Maxwell's equation of diff usion. ${ }^{2}$ It states that there acts on a particle of the first gas a force due to the pressure gradient of the first gas, and a force proportional to the difference of the velocities of the two gases.

The ordinary equation of diffusion, or Fick's law, ${ }^{2}$ was deduced by Stefan ${ }^{1}$ from (1) and the equation of continuity by assuming that $\xi_{1}$ was negligible.

We shall now assume that $u_{2}$ is negligible. This is the case in which the second gas is immobile and the first gas diffuses through it. Some problems involving two gases can be reduced to just such a problem."

If now in (1) we set $u_{1}=\eta, \xi_{1}=d v / d t, A_{12} \rho_{2}=a, p_{1}=p, \rho_{1}=\rho$, we obtain

$$
\frac{d v}{d t}=-\frac{1}{\rho} \frac{\partial p}{\partial x}-a v \text {. }
$$

The corresponding three dimensional form when there is present in addition a body force per unit mass represented by the vector $\mathbf{F}$ is

$$
\frac{d v}{d t}=\mathrm{F}-\frac{\nabla p}{p}-a \mathbf{v},
$$

where $\nabla$ is the velocity vector. This equation is the equation of motion of a viscous fluid with the viscosity terms replaced by $a$ force proportional to the velocity.

We shall now deduce some additional equations which are consequences of (2).

If we multiply (2) scalarly be an arbitrary virtual displacement $\delta e$, we obtain, since $\delta \equiv \nabla \cdot \delta$ e,

$$
\frac{d v}{d t} \cdot \delta e=F \cdot \delta e-\frac{\delta p}{\rho}-a v \cdot \delta e .
$$

* Received Feb. 8, 1944.

1 J. Stefan, Ber. der Wiener Akad. 63 (2), 63-124 (1871).

2 J. C. Maxwell, Phil. Mag. (4), 35, 185-217 (1868).

3. M. Barrer, Diff usion in and through solids, Cambridge University Press, Cambridge, 1941, p. 1.

B. Lewis and G. v. Elbe, Combustion flames and explosion of gases, Cambridge University Press, Cambridge, 1938, p. 224. 
When the expression $\mathbf{v} \cdot d(\delta \mathrm{e}) / d t=\delta\left(\frac{1}{2} v^{2}\right)$ is added to both sides of (3), we obtain

$$
\frac{d}{d t}(\mathbf{v} \cdot \delta \mathbf{e})=\mathbf{F} \cdot \delta \mathbf{e}-\frac{\delta p}{\rho}+\delta\left(\frac{1}{2} v^{2}\right)-a \mathbf{v} \cdot \delta \mathbf{e} .
$$

If now we set $\delta \mathbf{e}=d \mathbf{e}$, where $d \mathbf{e}$ follows the natural motion of the system, and assume that $\mathbf{F}$ has a potential $U$, then from (3)

or

$$
d\left(\frac{1}{2} v^{2}\right)=d U-\frac{d p}{\rho}-a \boldsymbol{\nabla} \cdot d \mathbf{e},
$$

$$
d\left(\frac{1}{2} v^{2}\right)+a v^{2} d t=d U-\frac{d p}{\rho} .
$$

If the temperature of the gas is constant, we have

$$
p=K \rho, \quad \int \frac{d p}{\rho}=K \ln p,
$$

$K$ being a constant. Integration of (6) then yields

$$
\frac{1}{2} v^{2}+K \ln p+a \int \nabla \cdot d e=\text { const. }
$$

When the only body force is due to gravity, and the $x$-axis is vertically downward, $U=g x$ and (7) becomes

$$
x-\frac{K}{g} \ln p-\frac{v^{2}}{2 g}-\frac{a}{g} \int \nabla \cdot d e=\text { const. }
$$

We now return to (3). If there is a straight or curved axis of symmetry such that, if $s$ is the arc length of this axis, $v, p, \rho$ are functions of $s, t$ only, and if we set $\delta \mathbf{e}=\mathbf{u}_{\varepsilon} \delta s$, where $u_{d}$ is a unit vector tangent to the axis of symmetry, then (3) becomes

or, since $\boldsymbol{v} \cdot \mathbf{u}_{\mathbf{s}}=\boldsymbol{v}$,

$$
\frac{d \nabla}{d t} \cdot \mathbf{u}_{\delta} \delta s=g \delta x-\frac{\delta p}{\rho}-a \nabla \cdot \mathbf{u}_{s} \delta s,
$$

$$
\frac{d v}{d t}=g \frac{\partial x}{\partial s}-\frac{1}{\rho} \frac{\partial p}{\partial s}-a v .
$$

Now

$$
d v=\frac{\partial v}{\partial s} d s+\frac{\partial v}{\partial t} d t, \quad \frac{d v}{d t}=\frac{\partial v}{\partial s} v+\frac{\partial v}{\partial t},
$$

whence (9) takes the form

$$
\frac{\partial x}{\partial s}-\frac{1}{\rho g} \frac{\partial p}{\partial s}=\frac{\partial}{\partial s}\left(\frac{v^{2}}{2 g}\right)+\frac{1}{g} \frac{\partial v}{\partial t}+\frac{a}{g} v .
$$

The equation of continuity is

$$
\Omega \frac{\partial \rho}{\partial t}+\frac{\partial}{\partial s}(\rho \Omega v)=0,
$$


where $\Omega$ is the area of the cross section. When the gas is at constant temperature, $p=K \rho$, and (10) and (11) become

$$
\begin{gathered}
\frac{\partial}{\partial s}\left(x-\frac{K}{g} \ln p\right)=\frac{\partial}{\partial s}\left(\frac{v^{2}}{2 g}\right)+\frac{1}{g} \frac{\partial v}{\partial t}+\frac{a}{g} v, \\
\Omega \frac{\partial p}{\partial t}+\frac{\partial}{\partial s}(p \Omega v)=0 .
\end{gathered}
$$

If the flow is steady, $\partial v / \partial t=0$ and (12) becomes

$$
x_{1}-x_{0}+\frac{K}{g} \ln \frac{p_{0}}{p_{1}}=\frac{v_{1}^{2}}{2 g}-\frac{v_{0}^{2}}{2 g}+\frac{a}{g} \int_{s_{0}}^{r_{1}} v d s,
$$

where the subscripts zero and one refer to two cross sections, both viewed at the same instant. We set $P=\frac{1}{2}\left(p_{0}+p_{1}\right), \pi=p_{0}-p_{1}, \alpha=\frac{1}{2} \pi / P$, whence

$$
\begin{aligned}
p_{0} & =P+\frac{1}{2} \pi=P(1+\alpha), \quad p_{1}=P-\frac{1}{2} \pi=P(1-\alpha), \\
\ln \frac{p_{0}}{p_{1}} & =\ln \frac{1+\alpha}{1-\alpha}=2\left(\alpha+\frac{1}{3} \alpha^{3}+\cdots\right)=2 \alpha\left(1+\frac{1}{3} \alpha^{2}+\cdots\right) .
\end{aligned}
$$

When the difference of pressure at these two cross sections is small, $\frac{1}{3} \alpha^{2}$ is much smaller than 1 and we have approximately $\ln \left(p_{0} / p_{1}\right)=2 \alpha=\pi / P$. If $w$ is the specific weight at pressure $P$, then

and (14) becomes

$$
P=K \rho=\frac{K w}{g}, \quad \frac{K}{g \rho}=\frac{1}{w}, \quad \frac{K}{g} \ln \frac{p_{0}}{p_{1}}=\frac{p_{0}-p_{1}}{w},
$$

$$
x_{1}-x_{0}+\frac{p_{0}-p_{1}}{w}=\frac{v_{1}^{2}}{2 g}-\frac{v_{0}^{2}}{2 g}+\frac{a}{g} \int_{s_{0}}^{s_{1}} v d s .
$$

Let us return once more to (2), and operate on it with $\nabla \times$ and $\nabla \cdot$, to obtain

$$
\begin{aligned}
& \frac{d}{d t}\left(\frac{\omega}{\rho}\right)=\left(\frac{\omega}{\rho} \cdot \Delta\right) \nabla-\frac{a}{\rho} \omega, \\
& \frac{d \theta}{d t}=\Delta \psi-a \theta-\nabla_{1} \cdot\left[\left(\nabla_{1} \cdot \nabla\right) \nabla\right],
\end{aligned}
$$

where $\omega=\nabla \times \nabla, \theta=\nabla \cdot \nabla, \psi=U-p / \rho, \rho$ is assumed to be a function of $p$ only, and

$$
\nabla_{1} \cdot\left[\left(\nabla_{1} \cdot \nabla\right) \mathbf{v}\right]=\theta^{2}-2 \sum \mathbf{k} \cdot\left(\frac{\partial \mathbf{v}}{\partial x} \times \frac{\partial \mathbf{v}}{\partial y}\right),
$$

$\mathbf{i}, \mathbf{j}, \mathbf{k}$ being unit vectors along the $x, y, z$-axes, respectively. The equation of continuity is

$$
\frac{d \rho}{d t}=-\rho \theta,
$$

or

$$
\frac{\partial \rho}{\partial t}=-\nabla \cdot(\rho \nabla)
$$


If $a$ is not a constant, but a function of $\mathrm{e}(x, y, z)$ for example, we must add the term $-(\nabla \times \nabla a) / \rho$ to the second term in (16).

We shall now examine the propagation of discontinuities in the boundary conditions, in the simple case of one dimension without gravity. Equations (2) and (18) become

$$
\begin{array}{r}
\frac{\partial v}{\partial t}+\frac{\partial v}{\partial x} v+\frac{1}{\rho} \frac{\partial \rho}{\partial x}+a v=0, \\
\frac{\partial \rho}{\partial t}+\frac{\partial \rho}{\partial x} v+\rho \frac{\partial v}{\partial x}=0 .
\end{array}
$$

If the temperature is constant, $p=K \rho$ and these equations become

$$
\begin{aligned}
& \frac{\partial v}{\partial t}+v \frac{\partial v}{\partial x}+\frac{K}{\rho} \frac{\partial \rho}{\partial x}=-a v, \\
& \frac{\partial \rho}{\partial t}+v \frac{\partial \rho}{\partial x}+\rho \frac{\partial v}{\partial x}=0,
\end{aligned}
$$

which are a system of two simultaneous quasilinear equations of the first order. These equations are usually referred to as Hamburger's equations in two dependent variables. By standard procedures, ${ }^{5}$ the ordinary differential equations for the characteristics are found in the form

$$
\begin{array}{ll}
\frac{d t}{d x}=\left(v+K^{1 / 2}\right)^{-1}, & d v+K^{1 / 2} \rho^{-1} d \rho=-a v\left(v+K^{1 / 2}\right)^{-1} d x, \\
\frac{d t}{d x}=\left(v-K^{1 / 2}\right)^{-1}, & d v-K^{1 / 2} \rho^{-1} d \rho=-a v\left(v-K^{1 / 2}\right)^{-1} d x .
\end{array}
$$

If $x, t, v$ are regarded as a rectangular cartesian coordinate system, and $x, t, \rho$ as a second system, the solutions of Eqs. (24) and (25) can be represented graphically as surfaces. We may assign boundary conditions as follows:

(a) In both the $x v$ - and the $x \rho$-planes, a curve is given.

(b) In the $t \rho$-plane, a curve is given; the corresponding curve in the $t v$-plane must be determined by means of the characteristics.

(c) In a plane $x=$ const. $=d$ of the $x t \rho$-system, a curve is given; the corresponding curve in the plane $x=d$ of the $x v t$-system must be determined by means of the characteristics.

Let us consider, for example, a gas for which $K=13.7 \times 10^{4} \mathrm{~m} .{ }^{2} \mathrm{sec}^{-{ }^{-2}, a}=2.75 \times 10^{9}$ sec. $^{-1}$, with the following boundary conditions:

(a) In the $x v$ - and $x \rho$-plane, $v=v_{0}=0, \rho=\rho_{0}=0.073 \mathrm{~kg} . \mathrm{m} .{ }^{-4} \mathrm{sec} .{ }^{2}$ This corresponds to an initial pressure at rest of $10000 \mathrm{~kg} \cdot \mathrm{m} .^{-2}$

(b) In the $t \rho$-plane, the curve $\rho=f(t)$ is given.

(c) In the plane $x=2000 \mathrm{~m}$. of the $x t \rho$-system, the curve $\rho=0$ is given.

When $v=0$, we find from the first of Eqs. (24) that $d t / d x=K^{-1 / 2}=370 \mathrm{~m} . \mathrm{sec}^{-1}$. Thus the time that the initial discontinuity requires to cover the distance $x=2000 \mathrm{~m}$. is $5.4 \mathrm{sec}$.

' A. R. Forsyth, Theory of differential equations, Cambridge University Press, 1906, vol. 5, p. 435. 